\title{
Mislocalisation of Pax7 and Its Interaction with Importin-a1 (KPNA2) in Dystrophin-Deficient Myoblasts
}

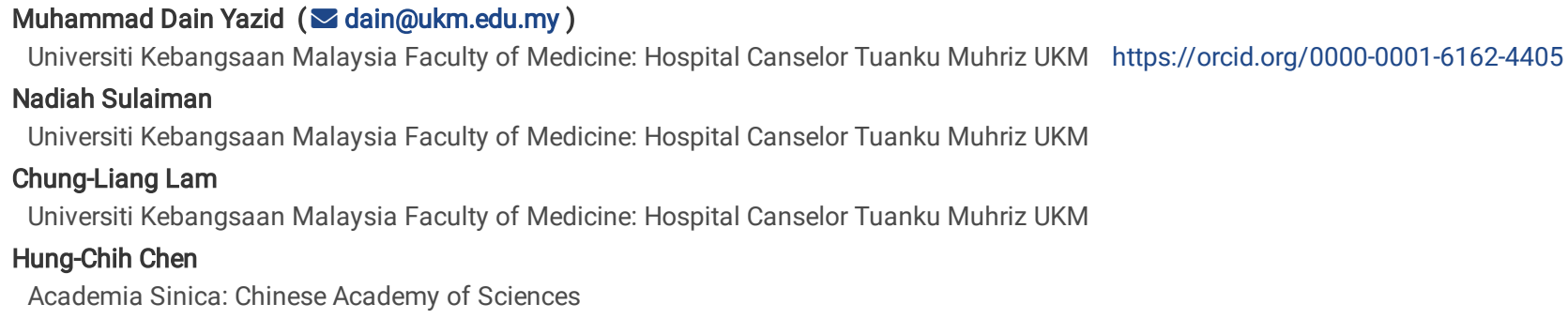

\section{Research}

Keywords: Pax7, KPNA2, dystrophin-deficient myoblast, Duchene muscular dystrophy

Posted Date: August 12th, 2021

DOI: https://doi.org/10.21203/rs.3.rs-786571/v1

License: (c) (i) This work is licensed under a Creative Commons Attribution 4.0 International License. Read Full License 


\section{Abstract}

Background: Pax 7 is one of the key factors in the development of tissues and organs during embryogenesis. It has been suggested that Pax7 may play a major role during myogenesis. Our previous study has shown that Pax7 cell is attenuated in the $m d x$ embryo during gestation as well as in dystrophic muscle indicating that an absence of dystrophin in muscle affects pax7 regulation in Duchene Muscular Dystrophy (DMD). Therefore, we aimed to investigate the Pax7 expression pattern as well as their specific transport protein in dystrophin-deficient myoblasts at postnatal/juvenile stage.

Methods: In this study, dfd13 (dystrophin-deficient) and C2C12 (non-dystrophic) myoblasts were cultured under normal conditions prior to further analyse its expression pattern at proliferating stage via western blot and immunofluorescence analysis. Protein prediction and protein interaction study was done via in silico and co-immunoprecipitation analyses, respectively.

Results: It was found that Pax7 localised in the cytoplasm of dystrophin-deficient myoblasts and high expression retained during differentiation. Colocalisation analysis of Pax7 with subcellular markers indicated that Pax7 is highly synthesised at their proliferative state. Interestingly, it is shown that Pax7 possess a nuclear location signal and KPNA2 was suggested as an escort protein for Pax7 translocation into the nucleus.

Conclusion: For the first time, our study showed that Pax7 is mislocalised in dystrophin-deficient myoblasts and it is postulated that KPNA2 is the karyopherina which might be responsible for Pax7 translocation into the nucleus.

\subsection{Background}

Pax proteins are the key factors in the development of tissues and organs during embryogenesis. These proteins are characterised by the presence of a paired domain (PD), which specifically binds to DNA sequences where it acts as a transcription repressor or activator, and is generally located in the N-terminal part. Pax genes may also have an octapeptide (OP) motif and a homeobox DNA-binding domain (homeodomain; HD). Pax genes can be categorised into four subfamilies according to their structural characteristics. Within these subfamilies there is some overlap in expression during the development of tissues or organs in determining the choice of cell fate (1)

Pax7 is expressed specifically during the development of the nervous and muscular system. A study by Merrick et al. (2009) showed that Pax7 can be detected as early as E11.5 in wild-type embryos and its level increases throughout the gestational period (2). It plays a role in the regulation of muscle precursor cell proliferation by recruiting the histone-3-lysine-4 methyltransferase (H3K4 HMT) complex via Pax3/7BP and the Wdr5 adaptor. This complex then target genes, i.e. $C d c 20$ and $I d 3$, which are involved in cell-division cycle activation and the transcription inhibition process, respectively (3). The downregulation of pax7 protein levels in an embryo correlates with an increased level of apoptosis in myoblasts isolated from $\mathrm{mdx}$ and $\mathrm{cav}^{-/-}$mice (2).

Further observations have shown the attrition of a Pax7-positive cell population in $m d x$ and cav3 ${ }^{-/-}$embryos until late in gestation. Pax7-positive cells fragments were found in dystrophic muscle (E15.5: $\mathrm{cav3}^{-/-}$and $m d x$, E17.5: $m d x$ ) and was much more severe in $m d x / \mathrm{cav}^{+/-}$, suggesting that these cells may undergo apoptosis (2). From the results, the severity of the double mutant showed that dystrophin and cav-3 play an important role for the survival of Pax7positive cells during myogenesis. However, in vitro analysis of apoptosis in dystrophin-deficient myoblasts is lacking, which would demonstrate the frequency of apoptosis, as well as the significance of the event.

Recently, it has been reported that Pax7 is regulated/modified by SUMOylation. Pax7 interacts with the SUMO conjugating enzyme known as UBC9, which SUMOylates Pax7 at lysine85 (K85) within the DNA binding domain (4). SUMOylated-Pax7 is essential during neural crest development, C2C12 myogenic differentiation, and transcriptional transactivation. In adult satellite cells, the arginine methyltransferase Carm1, specifically methylates an arginine present in the N-terminus of Pax7, and thus is recruited to the WDR5-ASH2L-MLL2 complex for up-regulating Pax7 target genes (5). Pax3/7 have also been shown to interact with other proteins involved in chromatin binding, including HIRA, DAXX and PAX3/7BP (6). This binding is important for the up-regulation of Pax7 target genes, such as $I d 3$ and $C d c 20$. Id 3 is a helix-loop-helix $(\mathrm{HLH})$ protein that can also bind with other HLH proteins as a heterodimer. The lack of a basic DNA-binding domain inhibits other HLH proteins from binding to DNA, thus acting as a transcription inhibitor. Binding of Pax7 to the Id3 promoter is also thought to up-regulate and increase its expression during differentiation. Cdc20 is a regulatory protein that interacts with other proteins during cell division and is highly expressed in proliferating cells. It is responsible for mediating the association of the anaphase protein complex with the mitotic spindle checkpoint protein, MAD1L1 (7).

Different from prokaryotic cells, the nucleus and cytoplasm are separated by a double lipid bilayer nuclear envelope (NE) in eukaryotic cells. The nuclear pore complex (NPC), in the NE, tightly mediates the bidirectional traffic of molecules, including proteins, nucleic acids and small molecules, via aqueous channel either passively or actively $(8,9)$. The nuclear-cytoplasmic machinery involves the NPC, cellular apoptosis susceptibility (CAS), RanGTP, karyopherins (also known as importins/exportins) as well as the specific proteins/RNA complex to be transported. Alterations to any of these factors can cause the failure of protein regulation within a cell, thus affecting the whole process, i.e. up-regulation or down-regulation of genes.

The regulation of translated proteins in the cytoplasm is control by various signalling mechanisms, either for secretion or to remain within a cell. For those proteins that play a role as an activator, adaptor or gene regulator, specific transcription factors are need to already be present in the nucleus for the upregulation, as well as down-regulation of genes, thus they need to be transported into the nucleus. As most of these proteins are larger than $40 \mathrm{kDa}$, protein adaptors ( $\beta$-karyopherins) are needed to shuttle them through the nuclear membrane.

Transport receptors, also known as $\beta$-karyopherins (importins/exportins), only recognise proteins containing a nuclear localisation signal (NLS). This is a sequence highly enriched with the basic amino acids lysine $(K)$, arginine $(R)$ and histidine $(H)$. It has been reported that phosphorylation of the NLS enhances the binding affinity of importin- $a$ to become part of a complex (10), which then binds to importin- $\beta$ and is translocated into the nucleus via the NPC as the 
karyopherin- $\beta$ has a binding affinity for nucleoporins within the NPC. This translocation process is also regulated by the gradient of RanGTP, which is asymmetrically distributed and predominantly found in the nucleus. Dissociation of the NLS-protein complex by RanGTP occurs within the nucleus, where the concentration of RanGTP is controlled by RanGEF which converts RanGDP to RanGTP. Importin- $\beta$ is carried out of the nucleus by RanGTP, while importin- $\alpha$ needs a protein receptor (CAS) to form a complex with in order for RanGTP to be recycled back into the cytoplasm (8).

Earlier, our group has reported that Pax7 cell is attenuated in the $m d x$ embryo during gestation. Pax7 cell fragments have also been found in dystrophic muscle, indicating that the cells are undergoing apoptosis. Immunoblot analysis of whole embryos showed that Pax7 expression was also substantially reduced with age (2). This finding has led to the suggestion that dysregulation of Pax7 takes place in MD as early as during the embryonic stages. Therefore, in this study the Pax7 expression pattern has been further examined during the postnatal/juvenile stage in Pax7 myoblasts isolated from a 5-week old $m d x$ mouse.

As a transcription factor, Pax7 is mainly found in the nucleus of myoblasts, but surprisingly, it has been found not only in the nucleus, but also found in cytoplasmic region (i.e. organelles) of dystrophin-deficient myoblasts. However, there are a lack of studies to show what actually happens to Pax7 in dystrophin-deficient myoblasts. There may be a problem in protein trafficking in or out of the nucleus which involves the importin and exportin mediated pathway, or there could be an alteration in the NLS which causes the failure of Pax7 to bind to importins. This could be the cause of the failure of these cells to be differentiated into mature myotubes prior to the development of muscle tissue. Initially, the expression of Pax7 upon differentiation was determined. Furthermore, the cytoplasmic-Pax7 subcellular localisation, i.e. endoplasmic reticulum, golgi, recycling endosome and lysosome, for protein synthesis and degradation was examined using subcellular markers. Finally, protein prediction tools were utilised which suggested that a Pax7-karyopherin protein interaction is responsible for nuclear localisation.

\subsection{Materials And Methods}

\subsection{Cell culture and differentiation}

The $\mathrm{C} 2 \mathrm{C} 12$ myoblast cell line was used in this study, and was established from an adult mouse myoblast $\mathrm{C} 2$ cell line derived from the thigh muscle of a 2month-old mice. The dfd13 cell line was derived from a 5-week-old $m d x$ mouse $(11,12)$. Mouse embryonic fibroblast (MEF) cells were a gift from Adil Rashid, University of Birmingham, and were used as a control for the phosphorylation of Akt at threonine-308. Both myoblasts were maintained in growth medium (GM) consist of Dulbecco's Modified Eagles Medium (DMEM) (Invitrogen Ltd, UK) supplemented with 10\% Fetal Bovine Serum (FBS) (Sigma-Aldrich, UK), 1\% penicillin/streptomycin (Sigma-Aldrich, UK) and 1\% L-Glutamine (Sigma-Aldrich, UK) before plated for differentiation. To differentiate myoblasts, GM was replaced with differentiation medium (DM) which is DMEM supplemented with $2 \%$ Horse Serum (Sigma-Aldrich, UK), $1 \%$ penicillin/streptomycin (SigmaAldrich, UK) and $1 \%$ L-Glutamine and cultured for 10 days.

\subsection{Total protein extraction \& Protein sub-fractionation}

Protein was extracted from cells cultured in a $10 \mathrm{~cm}$ dish. The medium was removed and the cells washed twice with PBS before adding approximately 300 $\mu \mathrm{L}$ of lysis buffer ( $0.5 \%$ triton X-100, $0.5 \%$ deoxycholic acid; $0.5 \mathrm{M} \mathrm{NaCL} ; 0.02 \mathrm{M}$ Tris; $0.01 \mathrm{M}$ EDTA, pH 7.5) containing a protease inhibitor (complete ULTRA tablets, EDTA-free, protease inhibitor cocktail, Roche, UK). The cells were scrapped and collected into a tube before being subjected to centrifugation at 14000 rpm for 15 minutes at $4^{\circ} \mathrm{C}$. The pellet was discarded and the supernatant transferred to a new tube and stored at $-20^{\circ} \mathrm{C}$.

The rapid efficient and practical (REAP) protocol was used for protein sub-fractionation (13). All reagents were chilled and kept on ice all times. The medium was removed and the cells washed three times with PBS before adding approximately $1 \mathrm{~mL}$ of ice-cold PBS. The cells were scrapped and collected into a new tube and pop-spun for 10 seconds and the supernatant discarded. The pellet was resuspended in $900 \mu \mathrm{L}$ of ice-cold PBS containing $0.1 \%$ nonyl-

phenoxypolyethoxylethanol (NP-40) and approximately $300 \mu \mathrm{L}$ was removed to a new tube labelled as whole cell lysate (WCL). The remaining cells were popspun for 10 seconds and approximately $300 \mu \mathrm{L}$ was transferred to a new tube labelled as the cytosolic fraction, while the remainder of the supernatant was discarded. The pellet was washed with $1 \mathrm{~mL}$ of PBS containing $0.1 \%$ NP-40) and pop-spun for 10 seconds; this step was repeated 2 to 5 times to eliminate the cytosolic fraction. After washing was completed this was considered to be the nuclear fraction. The cytosolic fraction was also pop-spun 3 times to separate the remaining nuclear fraction. Both the WCL and nuclear fraction were sonicated three times for 10 seconds and boiled for 1 minute.

\subsection{Immunoprecipitation Assay}

In this study, Pax7 and importin13 were immunoprecipitated using a protein A sepharose CL-4B bead slurry. KPNA2 were immunoprecipitated using Dynabeads ${ }^{\circledR}$ Protein $\mathrm{G}$ magnetic beads.

\subsubsection{Immunoprecipitation using a Protein A Sepharose CL-4B Beads Slurry}

Approximately $100 \mu \mathrm{g}$ of cell lysate was diluted with lysis buffer and about $20 \mu \mathrm{L}$ of the protein A sepharose CL-4B beads slurry added to the lysate. The mixture was subjected to centrifugation at $2500 \mathrm{rpm}$ for 1 minute, and the pellet discarded. Approximately $1 \mu \mathrm{g}$ of antibody was added to the supernatant (precleared lysate) and incubated at $4^{\circ} \mathrm{C}$ for 2 hours on a rotator. Next, $30 \mu \mathrm{L}$ of the protein A Sepharose CL-4B beads slurry was added into the antibody-protein complex and incubated at $4^{\circ} \mathrm{C}$ overnight. The mixture containing the complex-bound beads was subjected to centrifugation at $2500 \mathrm{rpm}$ for 1 minute. The

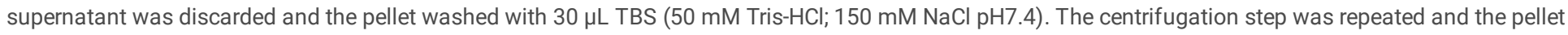
resuspended in $20 \mu \mathrm{L}$ sample buffer and heat-denatured at $95^{\circ} \mathrm{C}$ for 7 minutes. The mixture was subjected to centrifugation at $13000 \mathrm{rpm}$ for $1 \mathrm{minute}$ and the supernatant used in Western blot analysis.

\subsubsection{Dynabeads@ Protein G Magnetic Beads}

Page 3/14 
Dynabeads ${ }^{\circledR}$ Protein $\mathrm{G}$ magnetic beads were vortexed for about 30 seconds and approximately $50 \mu \mathrm{L}$ of Dynabeads transferred to a new tube and placed on a magnet to separate the beads from the solution. The supernatant was removed and antibody diluted in $200 \mu \mathrm{L}$ PBST (PBS containing $0.02 \%$ Tween 20 ) added, before being were incubated with rotation at room temperature for 10 minutes. The tube was placed on a magnet for bead separation and washed with $400 \mu \mathrm{L}$ PBST. Approximately $500 \mu \mathrm{g}$ cell lysate was added to the beads and the mixture incubated with rotation for 30 minutes at room temperature. Next, the tube was placed on a magnet and the supernatant discarded before washing the beads three times with ice-cold PBST. About $20 \mu \mathrm{L}$ of sample buffer was added to the cleaned beads which were resuspended before being boiled at $95^{\circ} \mathrm{C}$ for 7 minutes.

\subsection{Antibody Crosslinking}

Antibody crosslinking was performed to avoid non-specific binding of antibodies raised is the same host. The crosslinking step was performed at the beginning of immunoprecipitation procedure. After the Dynabeads had been incubated with the antibody for 10 minutes, the tube was placed on a magnet for bead-solution separation. The supernatant was discarded and the beads washed with PBST, followed by two washes in washing buffer ( $0.2 \mathrm{M}$ triethanolamine, TEA; Sigma-Aldrich, UK, in PBS). The antibody-bound beads were resuspended in crosslinking buffer (20 mM dimethyl pimelidate dihydrochloride (DMP; Sigma-Aldrich, UK, in wash buffer) and incubated at room temperature for 30 minutes on a rotator. The tube was placed on a magnet for bead-solution separation and the supernatant discarded. The beads were washed three times with quenching buffer $(0.05 \mathrm{M}$ tris- $\mathrm{HCl} \mathrm{pH} 7.5)$ for 15 minutes on a rotator before approximately $500 \mu \mathrm{g}$ of cell lysate (diluted in lysis buffer up to $1 \mathrm{~mL}$ ) was added, and then incubated at room temperature for 30 minutes on a rotator. The tube containing the Dynabeads-Ab-antigen complex was washed three times with ice-cold PBS and the complex resuspended in sample buffer and boiled for 7 minutes at $95^{\circ} \mathrm{C}$ prior to Western blot analysis.

\subsection{Western blotting}

Equal protein loading was prepared before resuspend with $2 \times$ Laemmli sample buffer. The mixture was collected by short spin centrifugation and boiled for 5 minutes before loaded into SDS-polyacrylamide gel electrophoresis. Lysate were separated on SDS-polyacrylamide gel electrophoresis (6, 8 or 15\% acrylamide) with $200 \mathrm{~V}$ for 1 hour. The separated protein were transferred onto nitrocellulose membrane using Transblot Turbo® Transfer System (BioRad, UK). The membranes were blocked in 5\% reduced fat milk for 1 hour prior to incubate with primary antibodies. The antibodies are a-tubulin, SSRP-1 (1:1000) from Sigma-Aldrich, UK.

\subsection{Immunoflourescence}

The cover slips were acid-etched for 5 minutes with nitric acid. This will help myoblasts firmly attached onto the cover slips prior for immunofluorescence. It has been sent for sterilisation before myoblasts were seeded onto it. The myoblasts/myotubes were fixed using $4 \%$ PFA. After fixation cells were permeabilised with $0.25 \%$ triton X-100 for 5 minutes to allow the antibody to bind specifically. The cells were then washed three times for 5 minutes with PBS before blocking with $5 \%$ BSA at room temperature for 30 minutes. The blocking solution was removed and the cells incubated with the primary antibodies at $4^{\circ} \mathrm{C}$ overnight. The primary antibody was removed the next day and the cells washed before probed using a secondary antibody (biotinylated) for 1 hour at room temperature. After washing, the cells were incubated with streptavidin-Texas red (1:1000) at room temperature for 1 hour in the dark. The cells were then counterstained with DAPI (1:100) and mounted onto slides using DakoCytomation. The slides were wrapped in aluminium foil and stored at $4^{\circ} \mathrm{C}$.

\subsection{Microscopy}

Images were taken using a confocal microscope (Nikon A1R) under 60x magnification and were analysed/edited using the NIS element software provided by Nikon Corporation. The images were analysed using NIS-elements provided by Nikon $®$ Instruments using object based overlap analysis (ROI). The image was first selected using ROI properties and then separated into a monochrome image and converted into a binary image prior to the threshold. Each binary image was processed using a binary operation to create binary overlays that only exist when the green and red channel signals overlap. Each analysis was repeated at least three times for different samples obtained at different times/experiment.

\subsection{Statistical Analysis}

All Western blots were repeated at least three times for different protein samples obtained at different times/experiment. Statistical analyses were carried out using the student t-test (Microsoft Excel) and differences were considered significant at $p<0.05$ and $p<0.01$. Standard error was obtained using analysis of variance (ANOVA) in Microsoft excel.

\subsection{Results}

\subsection{Pax7 Expression Remains High in Differentiating Dystrophin-Deficient Myoblasts}

As Pax7 cells and Pax7 expression was found to be attenuated in $m d x$ embryos, its expression in the postnatal/juvenile stage was investigated. C2C12 and dfd13 myoblasts were initially cultured in GM overnight or until they reached $90 \%$ confluence before being replaced with DM. The cells were harvested and subjected to whole protein extraction for ND (non-differentiated) and after 4, 7 and 10 days of differentiation. Each sample was immunoblotted with an antiPax7 antibody to examine the level of Pax7 expression throughout the 10 days of differentiation.

Immunoblotting (Fig. 1) showed that Pax7 is highly expressed in both types of undifferentiated myoblasts, although slightly lower in dfd13 myoblasts. Densitometry analysis showed that the level of Pax7 expression in differentiating dfd13 myoblasts was higher than in differentiating C2C12 myoblasts throughout the 10 days of differentiation. The level of Pax7 showed a significant reduction at day 4 and day 7 and was slightly increased at day 10 in the myogenic differentiation of $\mathrm{C} 2 \mathrm{C} 12$ cells when compared to the non-differentiated state. However, Pax7 expression in differentiating dfd13 myoblasts remained high until day 7 and had decreased by day 10 . There was a significant difference in Pax 7 expression between both the differentiating C2C12 and dfd13 myoblasts on day 7. 
Pax7 is a transcription factor that plays role in gene regulation/activation during myogenesis. It has been reported that Pax7 directly regulates Myf5 (14,15) however, up-regulation of Pax7 expression inhibits myogenic differentiation due to the down-regulation of myogenin $(16,17)$, and differentiation onset is delayed. In this study, Pax7 remained at a high level of expression 7 days after differentiation commenced, indicating that differentiation may be delayed in dfd13 myoblasts. However, there was a significant reduction in Pax7 on day 10 in dfd13 myoblasts, suggesting that dfd13 myoblasts are prone to apoptosis. Pax7 acts as a survival factor in myotubes $(16,17)$, thus down-regulation of Pax7 may cause apoptosis.

\subsection{Pax7 is Mislocalised in Proliferating Dystrophin-Deficient Myoblasts}

To examine the Pax7 expression pattern, both types of myoblasts were subjected to immunofluorescence and sub-fractionation analyses. There is a lack of studies on Pax7 regulation in myoblasts in terms of the post-translational modifications, i.e. phosphorylation events, as well as protein distribution. In this study, Pax7 expression and its distribution was assessed in dystrophin-deficient myoblasts, with C2C12 myoblasts used as a control.

In line with the role of Pax7 as a transcription factor, the immunofluorescence data showed the localisation of Pax7 within the nucleus of both types of myoblasts (Fig. 2). However, it also showed that Pax7 was localised outside the nucleus, within the cytoplasm compartment. The pattern of Pax7 distribution was mostly perinuclear, and in the dfd13 myoblasts it could also be found scattered within the cytoplasm (Fig. 2A). Pax7 was labelled with AlexaFlour $\AA_{594}$ dye (red) in both types of myoblasts and counterstained with DAPI. The images were taken using a confocal microscope (Nikon A1R) and were analysed/edited using the NIS element software provided by Nikon Corporation. However, this analysis was not able to provide the exact amount of Pax 7 expression in both compartments, thus immunoblotting analysis was performed.

Cellular fractionation was performed for both types of myoblast during the proliferative stage. For protein yield validation, specific markers were used, including the structure-specific recognition protein-1 (SSRP-1) and a-tubulin for nuclear and cytoplasmic fractions, respectively. Immunoblots showed that SSRP-1 was not present in the cytoplasmic fraction and a-tubulin was completely absent from the nuclear fraction, indicated that these fractions were not contaminated with each other (Fig. 2B).

Immunoblot analysis on the subfractionated myoblasts showed that about $27.5 \%$ of Pax 7 in $\mathrm{C} 2 \mathrm{C} 12$ cells was localised to the nucleus, while only $11.1 \%$ of Pax7 in dfd13 myoblasts was localised to the nucleus (Fig. 2B). Nuclear-Pax7 expression was normalised with SSRP-1, while cytoplasmic-Pax7 was normalised with a-tubulin. The immunoblot data revealed that most Pax7 was found in the cytoplasm of both types myoblast. The specific localisation distribution generally indicates that Pax7 is heading to an unknown protein event. For example, perinuclear Pax7 in C2C12 cells could be due to Pax7 being abundantly synthesised in the cytoplasm and then retained in the cell during the undifferentiated stage where it is maintained by cell proliferation.

Pax7 expression was found to be scattered throughout the cytoplasm of dfd13 myoblasts. This observation shows that Pax7 is extensively mislocalised in dfd13 myoblasts, suggesting that Pax7 is misregulated during the proliferative stage. Unpublished data from Chen (2013), showed that Pax7 was expressed at slightly higher levels in undifferentiated dfd13 myoblasts compared to undifferentiated C2C12 myoblasts. However, there no data has previously reported on the level of Pax7 expression in the nucleus and cytoplasm of myoblasts separately. Further examination of cytoplasmic-Pax7 in subcellular fraction was undertaken via co-localisation analysis.

\subsection{Pax7 May Undergo Protein Modification and Be Recycled in Proliferating Dystrophin- Deficient Myoblasts}

As indicated in Fig. 2, Pax7 was found to be expressed and more localised to the cytoplasm of dfd13 myoblasts compared to C2C12 myoblasts. To examine the exact location of Pax7 and identify the event that cytoplasmic-Pax7 might be involved in within proliferating dfd13 myoblasts, co-localisation of Pax7 with subcellular components, i.e. endoplasmic reticulum, golgi, recycling endosome and lysosome, was performed. Co-localisation analysis via immunofluorescence double labelling using specific markers for each location was undertaken and the subcellular markers utilised are listed in Table 1.

Table 1

Subcellular markers utilised

\begin{tabular}{|ll|}
\hline Subcellular Marker & Functions \\
\hline $\mathrm{Ca}^{2+}$ ATPase: Endoplasmic reticulum & $\begin{array}{l}\text { Protein is synthesised and prepared for sending to the Golgi prior to integral modification, sorting and } \\
\text { packaging. }\end{array}$ \\
\hline $\begin{array}{l}\text { Golgi Matrix protein-130kDa (GM130): } \\
\text { Golgi }\end{array}$ & Involved in maintaining the cis-Golgi structure. Protein is ready to be dispatched to other parts of the cell. \\
\hline Rab11: Recycling endosome & Transporting protein within the tubular network. \\
\hline CD107a: Lysosome & $\begin{array}{l}\text { Also known as lysosomal-associated membrane protein-1 (LAMP1), involved in the protein degradation } \\
\text { pathway. }\end{array}$ \\
\hline
\end{tabular}

As depicted in Fig. 3, Pax7 was labelled with Texas red (red) while the $\mathrm{Ca}^{2+}$ ATPase (ER) and GM130 (Golgi) markers were labelled with AlexaFlour ${ }^{8} 488$ (green). From the images taken, it can be seen that Pax7 co-localised with $\mathrm{Ca}^{2+}{ }^{2}$ TPase and GM130 in both proliferating $\mathrm{C} 2 \mathrm{C} 12$ and dfd13 myoblasts (Fig. $3 \mathrm{~A}$ ). Based on the statistical co-localisation analysis, Pax7 was found to be co-localised with the ER and Golgi markers in proliferating C2C12 myoblasts and dfd13 myoblasts. The co-localisation of Pax7 with $\mathrm{Ca}^{2+} \mathrm{ATPase}$ demonstrates that $\mathrm{Pax} 7$ is synthesised abundantly in proliferative $\mathrm{C} 2 \mathrm{C} 12 \mathrm{myoblasts}$ in line with the expression levels detected previously (Fig. 1). $\mathrm{Ca}^{2+}$ ATPase is localised to the ER membrane and involved in transporting calcium ions from the cytosol into the ER (18). Co-localisation of Pax7 to $\mathrm{Ca}^{2+}$ ATPase corresponds to the current location of Pax7. It has been reported that Pax7 expression is crucial for the 
maintenance and self-renewal of myoblasts $(19,20)$. Maintaining Pax7 expression retains the transcriptional regulation of Pax7 for myogenesis and for downregulation at the onset of muscle differentiation $(21,22)$.

GM130 is a protein localised specifically at the cis-Golgi, where it faces the ER in order to receive biosynthetic output from the ER. Co-localisation of Pax7 with GM130 suggests two possible events. Pax7 which is not used may be packaged and ready to be despatched to the final destination, most probably to lysosomes for digestion. Alternatively, Pax7 may be ready to be despatched back to the ER and to be translocated to the nucleus for transcription purposes. As GM130 has been found to be involved in signal transduction regulating migration and cell polarisation via kinases $(23,24)$, it could be suggested that Pax7 is also involved in these events. Further analysis is therefore needed into the co-localisation of Pax7 with recycling endosomes and lysosomes.

Cytoplasmic-Pax7 in both proliferative $\mathrm{C} 2 \mathrm{C} 12$ and dfd13 myoblasts has been shown to localise to the Golgi, suggesting that Pax7 might be processed, packaged and ready to be translocated to another myoblast compartment. It was hypothesised that Pax 7 would be digested/lysed rather than secreted for an external use as it plays a role as a transcription factor within the nucleus. In addition, Pax7 does not possess a signal peptide directing it towards a secretory pathway. To test this hypothesis, co-localisation of Pax7 within cells components, i.e. recycling endosome and lysosome, was explored. Rab11 and CD107a markers were used for recycling endosomes and lyososomes, respectively.

Co-localisation of Pax7 with recycling endosomes (Rab11) could be clearly seen in both proliferative C2C12 and dfd13 myoblasts (Fig. 3B). Rab11 is a small Ras-like GTPase regulatory protein which has a regulatory role in most membrane-transport steps, i.e. recycling of perinuclear proteins, the plasma membrane and golgi compartment endosomes (25). It is localised to the endocytic recycling compartment and trans-Golgi network. As Pax7 was found to co-localise with Rab11 in the perinuclear region, it can be suggested that here it is in the process of being transported from the Golgi to the ER following modification.

The co-localisation of Pax7 with lysosomes (CD107a) gave a lower score in both types of myoblasts (Table 2). CD107a, also known as lysosomal-associated membrane protein-1 (LAMP-1), is a type I transmembrane protein that has been widely used as a lysosome marker (26). As Pax7 was not co-localised with CD107a, this suggests that degradation of Pax7 may not occur to a significant extent. This result suggests that cytoplasmic-Pax7 in proliferative dfd13 myoblasts is significantly active within recycling endosomes but not purposely for degradation.

Table 2

Analysis of Pax7 co-localisation with subcellular markers

\begin{tabular}{|c|c|c|c|}
\hline Stage/State of Myoblasts & Protein events with respect Subcellular markers & C2C12 myoblasts & dystrophin-deficient myoblasts \\
\hline \multirow[t]{6}{*}{ Proliferating } & Protein synthesis/modification & Co-localised & Co-localised \\
\hline & - Endoplasmic reticulum ( $\left.\mathrm{Ca}^{2+} \mathrm{ATPase}\right)$ & Co-localised & Co-localised \\
\hline & • Golgi (GM130) & & \\
\hline & Protein transportation/ degradation & Co-localised & Co-localised* \\
\hline & - Recycling endosome (Rab11) & Not co-localised & $\left(p=2.62 \times 10^{-2}\right)$ \\
\hline & • Lysosome (CD107a) & & Not co-localised \\
\hline
\end{tabular}

\subsection{Identification of the Nuclear Localisation of Pax7-KPNA2 Interaction}

Synthesised protein that needs to be translocated into the nucleus needs an importin- $a$ and importin- $\beta$ recognition sequence within its NLS in order to form a complex. However, it remains unknown as to which protein adaptor is involved in shuttling Pax7 through the nuclear membrane.

\subsubsection{In silico Inspection of the Pax7 Sequence using NES and NLS analysis}

Based on the previous results, cytoplasmic-Pax7 has been found to be localised at higher levels in dfd13 myoblasts compared to C2C12 myoblasts. It appears that cytoplasmic-Pax7 is either from the nucleus or remains in the cytoplasm following translation process. It was therefore hypothesised that there must be an impairment in the nuclear-cytoplasmic transportation for Pax7 or other associated-proteins. The Pax7 protein sequence was obtained from the UniProt database (www.uniprot.org) and analysed to ascertain whether a Nuclear Localsiation Signal (NLS) and/or Nuclear Export Signal (NES) was present.

The NetNES 1.1 server developed by the Center for Biological Sequence Analysis (CBS), Technical University of Denmark (http://www.cbs.dtu.dk/services/NetNES/) was used to predict the NES sequence in Pax7. The server predicts leucine-rich NES in eukaryotic proteins using combination of Hidden Markov Model (HMM) and Artificial Neural Network (ANN) scores in the prediction algorithm and verify the method on the most recently discovered NES (Fig. 4A) with 0.5 as a threshold. However, as the NES score was below the threshold, no residue is expected to participate in a NES.

The cNLS mapper is software for NLS predictions in protein sequences and was developed by Kasugi et al. (2009) to recognise specific NLS for the importin a $\beta$ pathway by calculating NLS scores (levels of NLS activities) (27). In this study, it was used to analyse the NLS residues in the Pax7 sequence. The Pax7 sequence was obtained from the UniProt database (www.uniprot.org) and loaded into the software with a cut-off score of 5.0 selected. The cut-off score indicates the strength of NLS activity, with higher activities at 10.0 and lower activities at 1.0, based on an experiment where the GUS-GFP reporter protein was fused to an NLS. Searching for a bipartite NLS with a long linker in the protein sequence was also performed by selecting the 'within terminal 60 -amino-acids region' option, as this would help to identify a structure-dependent bipartite NLS which may be located close to each other in the tertiary structure (http://nlsmapper.iab.keio.ac.jp/). The analysis showed that a predicted bipartite NLS was present in the Pax7 sequence, starting at position 134 until residue 220 , 
shown in red text in Fig. 4B. The scores were 5.8, 5.5 and 5.1, which means that Pax7 has intermediate NLS activities, indicating it could be localised within both the nucleus and cytoplasm.

Analysis of phosphorylation sites was achieved using the NetPhos 2.0 server developed by the Center for Biological Sequence Analysis (CBS), Technical University of Denmark (http://www.cbs.dtu.dk/services/NetNES/). This server produces predictions for serine, threonine and tyrosine phosphorylation sites in eukaryotic proteins. The analysis shows that there are seven predicted serine phosphorylation sites within the NLS sequence, but no threonine or tyrosine phosphorylation sites were found (Fig. 3.9B). These sites indicate the positions that have the highest probability for importin binding via a phosphorylation event.

\subsubsection{Prediction of Importin-a1 and Importin13 as Pax7 Translocator in Myoblasts}

There is a lack of studies on the interactions of Pax7 with proteins involved in it translocation to the nucleus. Since Pax7 was found to be more localised within the cytoplasm of dfd13 myoblasts, it was hypothesised that the translocation of Pax7 into the nucleus is impaired, resulting in more cytoplasmic-Pax7 in these myoblasts. Two candidate proteins were chosen that may form a complex with Pax7, karyopherin- $\alpha$ (KPNA2) and karyopherin- $\beta$ or importin13 (IP013).

Karyopherin a1 has been shown to be involved in myoblast proliferation and differentiation, as a knockdown via siRNA targeting KPNA2 reduced both events (28). A study by Kovac et al. (2000) confirmed that importin a1 interacts with Pax5 via a NLS located at amino acids 195-201 within the central domain (29). Therefore, it was hypothesised that importin a1 could also be the Pax7 escort protein enabling its translocation into the nucleus. The Pax 5 and Pax7 sequences were aligned, revealing that 4 out of the 8 residues were similar within the binding site, suggesting that importin a 1 could potentially interact with Pax7 (Fig. 5). The NLS of both Pax7 and Pax5 was also analysed using NucPred by Brameier et al. (2007), which showed that both have a similar predicted NLS, suggesting that both proteins have a nuclear role (Fig. 3.11) (30).

Based on the STRING (Search Tool for the Retrieval of Interacting Genes/Proteins) database, no study has reported Pax7 (Mus musculus) protein interactions. However, it has been reported that Pax6 and Pax3 interact with IP013, indicating that these are potential cargo proteins for IP013 (31). Thus, it was hypothesised that IP013 may also play a role as a translocator for Pax7.

\subsubsection{Endogenous Pax7 is Associated with Importin-a1 (KPNA2) But Not Importin13 (IP013) in Myoblasts}

The data has shown that cytoplasmic-Pax7 was found localised in the Golgi and recycling endosomes not in lysosomes. This provides evidence that high level expression of Pax7 in differentiating dfd13 myoblasts is recycled and not degraded, retaining its function during this stage. Therefore, it was suspected that Pax7 transportation regulation is impaired during proliferation and differentiation of dfd13 myoblasts. Further investigations were carried out to determine the functional interaction of Pax7 with importin-a1 (KPNA2) in myoblasts.

There is no data relating to the Pax7 nuclear transport protein (karyopherin) available; however, based on the analysis presented, it is predicted that Pax7 possesses a NLS that importin could bind to. Analysis of importin candidates could be a Pax7 protein partner, identified IP013 and KPNA2. Coimmunoprecipitation analysis showed that only KPNA2 associates with Pax7 (Fig. 6A). Therefore to confirm this interaction, co-localisation analysis was undertaken. Co-localisation analysis also showed that Pax7 and KPNA2 are associated, suggesting that these proteins are interacting (Fig. 6B).

\subsection{Discussion}

The localisation of specific proteins, particularly transcription factors can be used to elucidate their specific functions and enable characterisation. Therefore, determinating the localisation of Pax7 was crucial to distinguishing and identifying whether this protein was functioning. As Pax7 levels remain highly expressed upon differentiation in dystrophin-deficient myoblasts., it is indicated that overexpression of Pax7 inhibits myoblast differentiation by downregulating MyoD, thus preventing myogenin induction (21). Therefore, further analysis of Pax7 localisation and subcellular-localisation during the proliferating and differentiating state was performed. Our study demonstrated that Pax7 were distributed extensively in the cytoplasm and the distribution analysis revealed that it is in the ER, golgi and recycling endosome of dystrophin-deficient myoblasts. However, ROI analysis showed that only Pax7 with rab11 colocalisation was significantly higher $\left(p=2.6 \times 10^{-2}\right)$ in dfd13 myoblasts compared to C2C12 myoblasts. This indicates that Pax7 is actively transported across the cytoplasm, thus explaining the extensive dispersion expression within the cytoplasm of dystrophin-deficient myoblasts. Significant co-localisation was defined as the percentage of Pax7 co-localised with rab11 in dfd13 myoblasts compared to $\mathrm{C} 2 \mathrm{C} 12$ myoblasts via a binary overlay.

As Pax7 was found in ER, golgi and recycling endosomes of dystrophin-deficient myoblasts, it was hypothesised that the protein translocator karyopherin may be impaired. To date, no study has reported on karyopherin complexing with Pax7 for translocation into the nucleus. It was identified in this study that KPNA2 interacts with Pax7 in both types of myoblasts examined. However, there was no impairment in terms of Pax7 binding to KPNA2 as our study showed that endogenous Pax7 is associated with KPNA2 in both types of myoblast. KPNA2 is a member of the karyopherin-a family of proteins which function as protein transporters to the nucleus. A study by Hall et al. (2011) demonstrated that KPNA2 is important for myoblast proliferation (28). Therefore, it can be suggested that high expression levels of KPNA2 reflect Pax7 levels in line with the co-immunoprecipitation analysis which showed both proteins are associated. High level expression of Pax7 may also reflect high binding to $\beta$-catenin, which inhibits myoblast differentiation (32). From the data obtained, it can be seen that association of Pax7 with KPNA2 complex suggests a Nuclear Localisation Vehicle.

\subsection{Conclusions}


In this study, it has been demonstrated that Pax7 is mislocalised in dystrophin-deficient myoblasts. For the first time, it has been established that KPNA2 is the karyopherin-a which may be responsible for Pax7 translocation to the nucleus.

\subsection{List Of Abbreviation}

\begin{tabular}{|c|c|}
\hline ANN & Artificial Neural Network \\
\hline BSA & Bovine Serum Albumin \\
\hline BSA & Bovine serum albumin \\
\hline $\mathrm{CO}_{2}$ & Carbon dioxide \\
\hline DAPI & 4',6-diamidino-2-phenylindole \\
\hline DM & Differentiation Medium \\
\hline DMD & Duchenne Muscular Dystrophy \\
\hline DMEM & Dulbecco's Modified Eagle's medium \\
\hline DMP & dimethyl pimelidate dihydrochloride \\
\hline DNA & Deoxyribonucleic Acid \\
\hline EDTA & Ethylenediaminetetra Acetic Acid \\
\hline ER & Endoplasmic reticulum \\
\hline FBS & Fetal Bovine Serum \\
\hline GM & Growth Medium \\
\hline GM130 & Golgi matrix protein-130 \\
\hline H3K4 HMT & histone-3-lysine-4 methyltransferase \\
\hline HD & homeodomain \\
\hline $\mathrm{HLH}$ & helix-loop-helix \\
\hline HMM & Hidden Markov Model \\
\hline IP013 & importin13 \\
\hline KPNA2 & Karyopherin Subunit Alpha 2 \\
\hline MD & Muscular Dystrophy \\
\hline $\mathrm{NaCl}$ & sodium chloride \\
\hline NE & nuclear envelope \\
\hline NES & Nuclear export signal \\
\hline NLS & nuclear localisation signal \\
\hline NP-40 & nonyl-phenoxypolyethoxylethanol \\
\hline NP-40 & nonyl-phenoxypolyethoxylethanol \\
\hline NPC & nuclear pore complex \\
\hline $\mathrm{OP}$ & octapeptide \\
\hline PAGE & Polyacrylamide Gel Electrophoresis \\
\hline PBS & Phosphate-Buffered Saline \\
\hline PBST & PBS containing $0.02 \%$ Tween 20 \\
\hline PD & paired domain \\
\hline $\mathrm{PH}$ & plekstrin homolog \\
\hline REAP & rapid efficient and practical \\
\hline SSRP-1 & structure-specific recognition protein-1 \\
\hline STRING & Search Tool for the Retrieval of Interacting Genes/Proteins \\
\hline TEA & triethanolamine \\
\hline WCL & Whole cell lysate \\
\hline
\end{tabular}




\subsection{Declarations}

\subsection{Ethics approval and consent to participate}

Not applicable

\subsection{Consent for publication}

All authors read and approved the paper

\subsection{Availability of data and material}

The datasets used and/or analyzed during the study are available from the corresponding author

\subsection{Competing Interests}

The authors report no conflicts of interest. The authors alone are responsible for the content and writing of the paper.

\subsection{Funding}

This research was performed with the financial support of Universiti Kebangsaan Malaysia (GUP-2019-030) \& Majlis Amanah Rakyat (MARA).

\subsection{Authors' Contribution}

Design the experiments: MDY CH-C. Performed the experiments: LCL MDY. Analyzed the data: NS MDY. Wrote the paper: LCL NS CH-C MDY. All authors read and approved the final manuscript.

\subsection{Acknowledgments}

The authors would like to thank Dr. Janet Smith and Dr. Neil Hotchin for their guidance and advice during research work. Also thank Dr. Melissa Grant (School of Dentistry, University of Birmingham), Dr. Debbie Cunningham and Dr. Trushar Patel and Professor John K. Heath (University of Birmingham) for helpful comments and idea throughout the work. Thanks to Dr. Shabana Begum and Dr. Adil Rashid (University of Birmingham) for reagents supplements.

\subsection{References}

1. Buckingham M, Relaix F. The role of Pax genes in the development of tissues and organs: Pax3 and Pax7 regulate muscle progenitor cell functions. Annu Rev Cell Dev Biol. 2007;23:645-73.

2. Merrick D, Stadler LKJ, Larner D, Smith J. Muscular dystrophy begins early in embryonic development deriving from stem cell loss and disrupted skeletal muscle formation. Dis Model Mech [Internet]. 2009;2(7-8):374-88. Available from:

http://dmm.biologists.org/cgi/doi/10.1242/dmm.001008\%5Cnhttp://www.ncbi.nlm.nih.gov/pubmed/19535499\%5Cnhttp://www.pubmedcentral.nih.gov/ artid=PMC2707105

3. Diao Y, Guo X, Li Y, Sun K, Lu L, Jiang L, et al. Pax3/7BP is a Pax7- and Pax3-binding protein that regulates the proliferation of muscle precursor cells by an epigenetic mechanism. Cell Stem Cell [Internet]. 2012 Aug 3 [cited 2017 Mar 19];11(2):231-41. Available from:

http://www.ncbi.nlm.nih.gov/pubmed/22862948

4. Luan Z, Liu Y, Stuhlmiller TJ, Marquez J, García-Castro MI. SUMOylation of Pax7 is essential for neural crest and muscle development. Cell Mol Life Sci [Internet]. 2013;70(10):1793-806. Available from: http://dx.doi.org/10.1007/s00018-012-1220-1

5. Kawabe Y, Wang YX, McKinnell IW, Bedford MT, Rudnicki MA. Carm1 Regulates Pax7 Transcriptional Activity Through MLL1/2 Recruitment During Asymmetric Satellite Stem Cell Divisions. Cell Stem Cell [Internet]. 2012 Sep 7;11(3):333-45. Available from: http://www.ncbi.nlm.nih.gov/pmc/articles/PMC3438319/

6. Buckingham M, Relaix F. PAX3 and PAX7 as upstream regulators of myogenesis. In: Seminars in cell \& developmental biology. Elsevier; 2015 . p. 115-25.

7. Kim HJ, Jamart C, Deldicque L, An G-L, Lee YH, Kim CK, et al. Endoplasmic reticulum stress markers and ubiquitin-proteasome pathway activity in response to a 200-km run. Med Sci Sport Exerc. 2011;43(1):18-25.

8. Kau TR, Way JC, Silver PA. Nuclear transport and cancer: from mechanism to intervention. Nat Rev Cancer. 2004;4(2):106-17.

9. Wente SR, Rout MP. The nuclear pore complex and nuclear transport. Vol. 2, Cold Spring Harbor perspectives in biology. 2010.

10. Nardozzi JD, Lott K, Cingolani G. Phosphorylation meets nuclear import: a review. Cell Commun Signal [Internet]. 2010;8(1):32. Available from: http://www.biosignaling.com/content/8/1/32 
11. Smith J, Schofield PN. The effects of fibroblast growth factors in long-term primary culture of dystrophic (mdx) mouse muscle myoblasts. Exp Cell Res. 1994;210(1):86-93.

12. Smith J, Schofield PN. Stable integration of an mdx skeletal muscle cell line into dystrophic (mdx) skeletal muscle: evidence for stem cell status. Cell Growth Differ [Internet]. 1997;8(8):927-34. Available from: http://www.ncbi.nlm.nih.gov/entrez/query.fcgi? cmd=Retrieve \&db=PubMed\&dopt=Citation\&list_uids $=9269902$

13. Suzuki K, Bose P, Leong-Quong RYY, Fujita DJ, Riabowol K. REAP: A two minute cell fractionation method. BMC Res Notes [Internet]. 2010 Nov 10;3:294. Available from: http://www.ncbi.nlm.nih.gov/pmc/articles/PMC2993727/

14. McKinnell IW, Ishibashi J, Grand F Le, Punch VGJ, Addicks GC, Greenblatt JF, et al. Pax7 activates myogenic genes by recruitment of a histone methyltransferase complex. Nat Cell Biol [Internet]. 2008;10(1):77-84. Available from:

http://www.nature.com.ep.fjernadgang.kb.dk/ncb/journal/v10/n1/full/ncb1671.html\%5Cnhttp://www.nature.com.ep.fjernadgang.kb.dk/ncb/journal/v10/

15. Günther S, Kim J, Kostin S, Lepper C, Fan C-M, Braun T. Myf5-Positive Satellite Cells Contribute to Pax7-Dependent Long-Term Maintenance of Adult Muscle Stem Cells. Cell Stem Cell [Internet]. 2013 Nov 7;13(5):590-601. Available from: http://www.ncbi.nlm.nih.gov/pmc/articles/PMC4082715/

16. Olguin HC, Yang Z, Tapscott SJ, Olwin BB. Reciprocal inhibition between Pax7 and muscle regulatory factors modulates myogenic cell fate determination. J Cell Biol. 2007;177(5):769-79.

17. Olguín HC, Pisconti A. Marking the tempo for myogenesis: Pax7 and the regulation of muscle stem cell fate decisions. J Cell Mol Med [Internet]. 2011;114. Available from: http://www.ncbi.nlm.nih.gov/pubmed/21615681

18. Vandecaetsbeek I, Vangheluwe P, Raeymaekers L, Wuytack F, Vanoevelen J. The Ca2+ Pumps of the Endoplasmic Reticulum and Golgi Apparatus. Cold Spring Harb Perspect Biol. 2011;

19. Kuang S, Chargé SB, Seale P, Huh M, Rudnicki MA. Distinct roles for Pax7 and Pax3 in adult regenerative myogenesis. J Cell Biol [Internet]. 2006 Jan 2;172(1):103-13. Available from: http://www.ncbi.nlm.nih.gov/pmc/articles/PMC2063538/

20. Lepper C, Conway SJ, Fan C-M. Adult satellite cells and embryonic muscle progenitors have distinct genetic requirements. Nature. 2009;460(7255):62731.

21. Olguin HC, Olwin BB. Pax-7 up-regulation inhibits myogenesis and cell cycle progression in satellite cells: a potential mechanism for self-renewal. Dev Biol. 2004;275(2):375-88.

22. Florkowska A, Meszka I, Zawada M, Legutko D, Legutko D, Proszynski TJ, et al. Pax7 as molecular switch regulating early and advanced stages of myogenic mouse ESC differentiation in teratomas. Stem Cell Res Ther. 2020;

23. Preisinger C, Short B, De Corte V, Bruyneel E, Haas A, Kopajtich R, et al. YSK1 is activated by the Golgi matrix protein GM130 and plays a role in cell migration through its substrate 14-3-3?? J Cell Biol. 2004;164(7):1009-20.

24. Millarte V, Farhan H. The Golgi in Cell Migration: Regulation by Signal Transduction and Its Implications for Cancer Cell Metastasis. Sci World J. 2012;2012:1-11.

25. Hutagalung AH, Novick PJ. Role of Rab GTPases in membrane traffic and cell physiology. Physiological Reviews. 2011.

26. Saftig P, Klumperman J. Lysosome biogenesis and lysosomal membrane proteins: Trafficking meets function. Nature Reviews Molecular Cell Biology. 2009.

27. Kosugi S, Hasebe M, Tomita M, Yanagawa H. Systematic identification of cell cycle-dependent yeast nucleocytoplasmic shuttling proteins by prediction of composite motifs. Proc Natl Acad Sci. 2009;106(25):10171-6.

28. Hall MN, Griffin CA, Simionescu A, Corbett AH, Pavlath GK. Distinct roles for classical nuclear import receptors in the growth of multinucleated muscle cells. Dev Biol. 2011;357(1):248-58.

29. Kovac CR, Emelyanov A, Singh M, Ashouian N, Birshtein BK. BSAP (Pax5)-importin a1 (Rch1) interaction identifies a nuclear localization sequence. J Biol Chem. 2000;275(22):16752-7.

30. Brameier M, Krings A, MacCallum RM. NucPred-predicting nuclear localization of proteins. Bioinformatics [Internet]. 2007;23(9):1159-60. Available from: http://www.ncbi.nlm.nih.gov/pubmed/17332022

31. Ploski JE, Shamsher MK, Radu A. Paired-type homeodomain transcription factors are imported into the nucleus by karyopherin 13. Mol Cell Biol. 2004;24(11):4824-34.

32. Zhuang L, Hulin JA, Gromova A, Tran Nguyen TD, Yu RT, Liddle C, et al. Barx2 and Pax7 have antagonistic functions in regulation of Wnt signaling and satellite cell differentiation. Stem Cells. 2014;32(6):1661-73.

\section{Figures}




\section{ND D4 D7 D10 ND D4 D7 D10}

$\operatorname{Pax} 7$

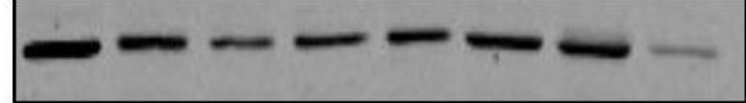

$\alpha$-tubulin
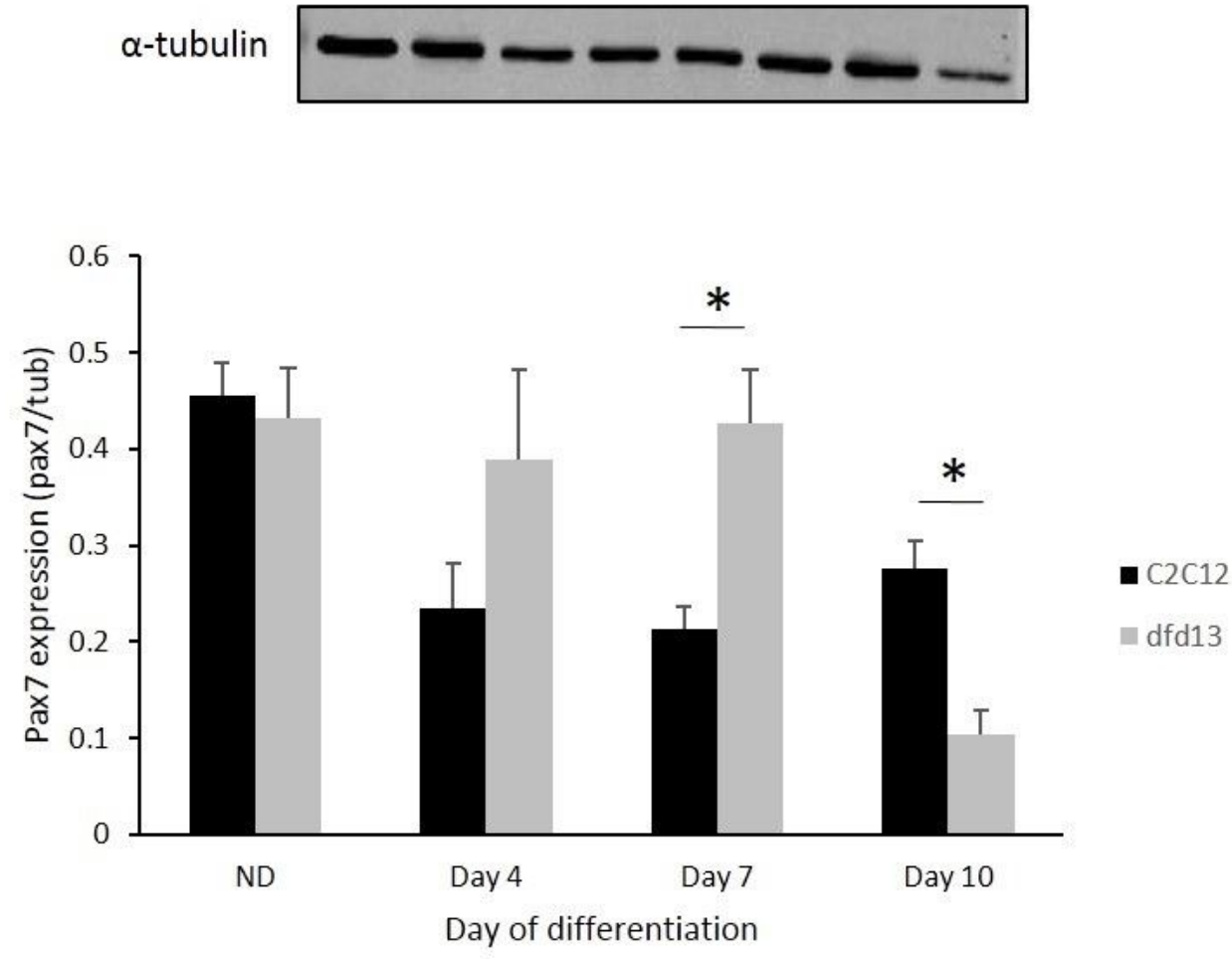

Figure 1

Pax7 expression remains high in differentiating myoblasts Both $\mathrm{C} 2 \mathrm{C} 12$ and dfd13 myoblasts were cultured in GM overnight. After reaching $90 \%$ confluence, myoblasts were washed with PBS and cultured for 10 days in DM which was changed every two days. Myoblasts were harvested at days 4,7 and 10 and subjected to protein extraction prior to immunoblotting with an antibody recognising Pax7, with a-tubulin used as a loading control. (A) Representative immunoblot of Pax7 and a-tubulin expression. (B) Densitometry analysis of Pax7 expression. The graph represents an average of three repeats from different samples. ND: non-differentiated; Significant different: * $(\mathrm{p}<0.05)$ compared to C2C12 myoblasts. GM: growth medium (DMEM + 10\% FCS); DM: differentiation medium (DMEM + 2\% horse serum); PBS: phosphate-buffered saline; $n=3$. 

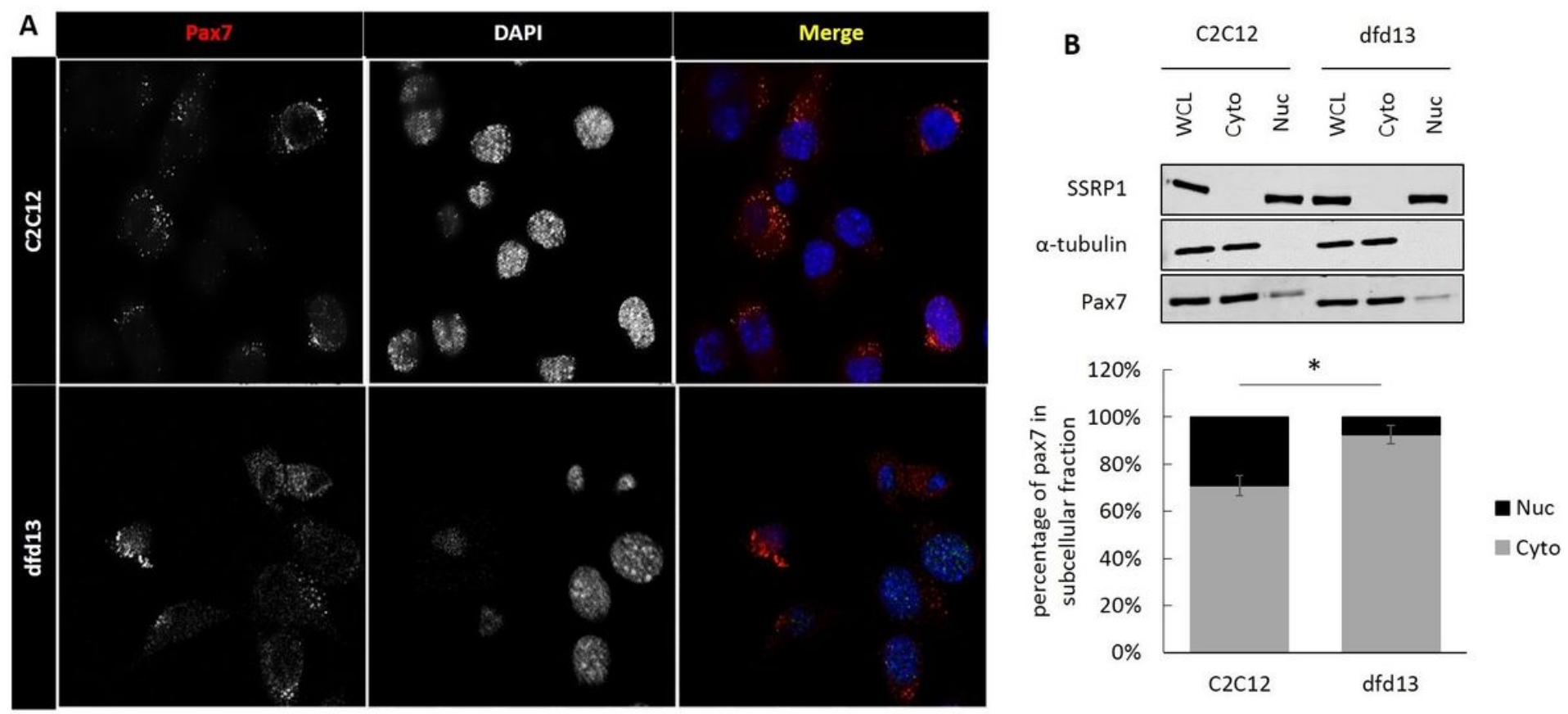

Figure 2

Pax7 is mislocalised in the cytoplasm of dystrophin-deficient myoblasts (A) Approximately $1.5 \times 104$ C2C12 and dfd13 myoblasts were cultured in GM until $60-70 \%$ confluent in a 48-well plate. Both types of myoblast were fixed in $4 \%$ PFA and subjected to immunofluorescence labelling. Pax7 was labelled with AlexaFlour594 and the myoblasts were counterstained with DAPI. Immunofluorescence of Pax 7 in $\mathrm{C} 2 \mathrm{C} 12$ and dfd13 images were taken using a confocal microscope (Nikon A1R) and were analysed/edited using the NIS element software provided by Nikon Corporation. (B) Both C2C12 and dfd13 myoblasts were cultured in GM and when 70\% confluent were harvested and subjected to sub-fractionation prior to immunoblotting with an anti-Pax7 antibody. SSRP-1 and a-tubulin were used as nuclear and cytoplasmic fraction control markers, respectively. Immunoblot for Pax7 expression and densitometry analysis of Pax 7 expression in both types of myoblasts as presented in (B). GM: growth medium (DMEM + 10\% FCS). WCL: whole cell lysate; Cyto: cytoplasmic; Nuc: nuclear; $n=3$.

A

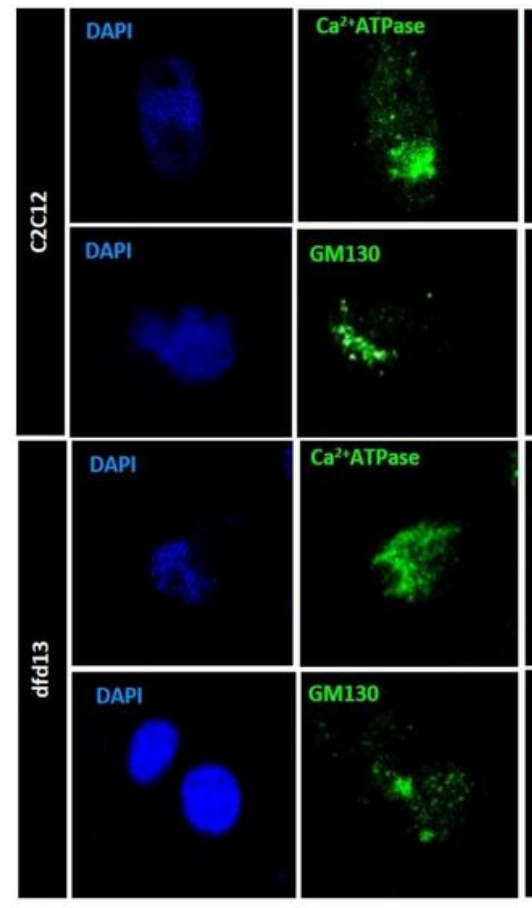

B

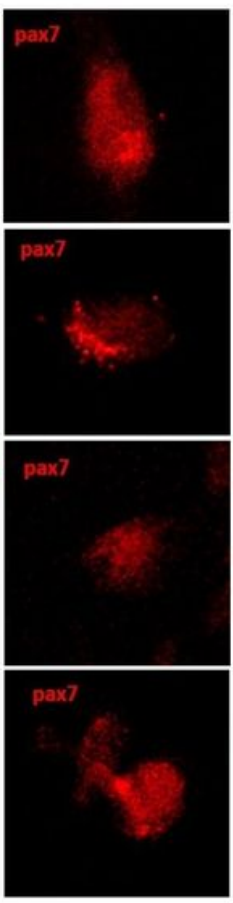

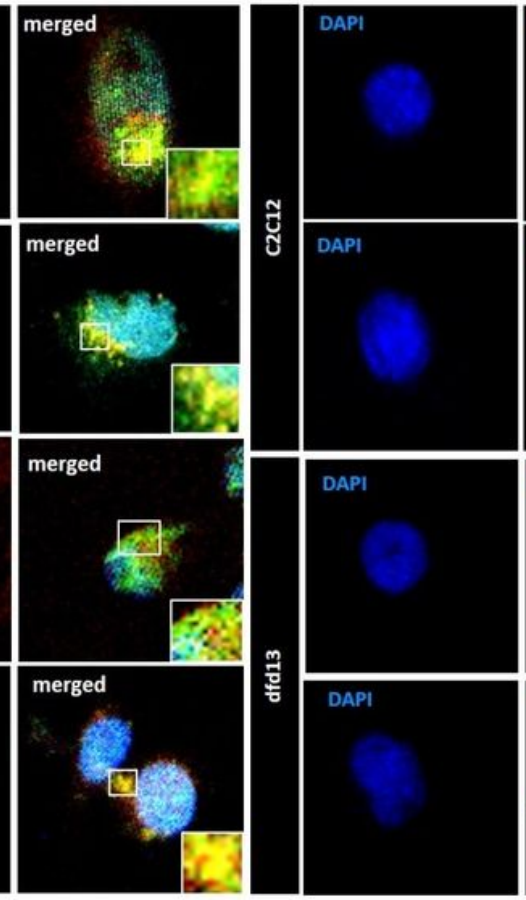

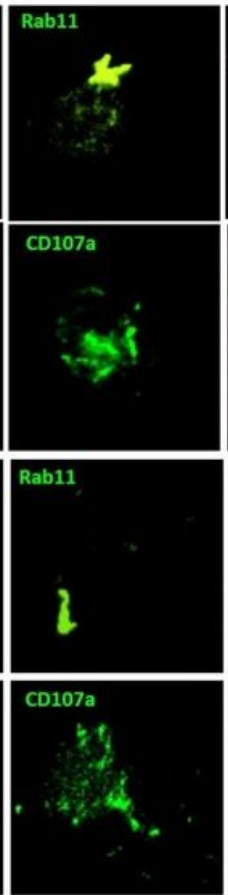

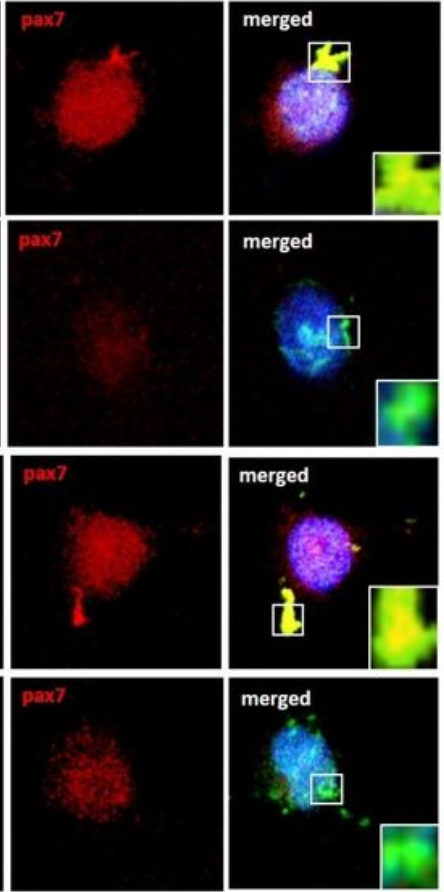

Figure 3

Cytoplasmic-Pax7 May Undergo Protein Modification and Be Recycled in Proliferating Dystrophin-Deficient Myoblasts Approximately 1.5 x 104 C2C12 (nondystrophic) and dfd13 (dystrophin-deficient) myoblasts were cultured in GM until 60-70\% confluent in a 48-well plate. Both types of myoblast were fixed in 4\% 
PFA prior to double immunolabelling for Ca2+ATPase or GM130 (green) and Pax7 (red) in (A) and Rab11 or CD107a (green) and Pax7 (red) in (B). DAPI was used as a nuclear counterstain. Images were taken using a Nikon A1 confocal microscope and co-localisation analysis was performed using NIS element software provided by Nikon. GM: growth medium (DMEM + 10\% FCS); $n=3$

A

\section{NetNES 1.1 Server - prediction results}

Technical University of Denmark

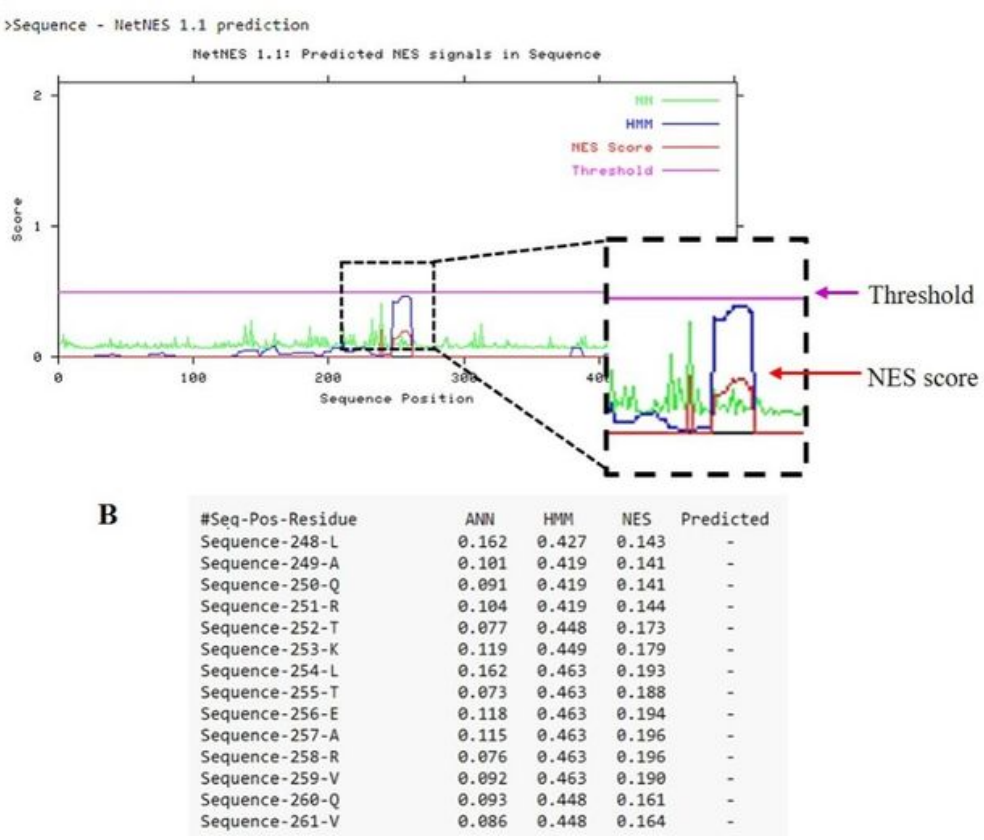

C

\begin{tabular}{|c|c|}
\hline Predicted NLSs in query sequence & \\
\hline MAALPGAVPRMMRPGPGQNYPRTGFPLEVSTPLGQGRVNQLGGVFINGRP 5 & \\
\hline LPNHIRHKIVEMAHHGIRPCVISRQLRVSHGCVSKILCRYQETGSIRPGA 1 & 100 \\
\hline IGGSKPRQVATPDVEKKIEEYKRENPGMFSWEIRDRLLKDGHCDRSTVPS 1 & \\
\hline VSSISRVLRIKFGKKEDDEEGDKKEEDGEKKAKHSIDGILGDKGNRLDEG 2 & 200 \\
\hline SDVESEPDLPLKRKQRRSRTTFTAEQLEELEKAFERTHYPDIYTREELAQ 2 & 250 \\
\hline RTKLTEARVQVWFSNRRARWRKQAGANQLAAFNHLLPGGFPPTGMPTLPP 3 & 300 \\
\hline YQLPDSTYPTTTISQDGGSTVHRPQPLPPSTMHQGGLAAAAAAADTSSAY 3 & 350 \\
\hline GARHSFSSYSDSFMNPGAPSNHMNPVSNGLSPQVMSILSNPSAVPPQPQA 4 & 400 \\
\hline DFSISPLHGGLDSASSISASCSQRADSIKPGDSLPTSQSYCPPTYSTTGY 4 & 450 \\
\hline SVDPVAGYQYSQYGQTAVDYLAKNVSLSTQRRMKLGEHSAVLGLLPVETG 5 & 500 \\
\hline QAY & 503 \\
\hline
\end{tabular}

\begin{tabular}{|c|c|c|}
\hline \multicolumn{3}{|c|}{ Predicted bipartite NLS } \\
\hline Pos. & Sequence & Score \\
\hline 134 & RDRLLKDGHCDRSTVPSVSSISRVLRIKFGKKE & 5.8 \\
156 & RVLRIKFGKKEDDEEGDKKEEDGEKKAKHSID & 5.5 \\
189 & ILGDKGNRLDEGSDVESEPDLPLKRKQRRSRT & 5.1 \\
\hline
\end{tabular}

D

Figure 4

NES and NLS analysis of the Pax7 sequence Pax7 has no potential sequence for exportin to bind to for its export out of the nucleus to the cytoplasm. (A) Graphic of the NES analysis showing that no residue scored over the threshold. (B) Some of the residues analysed by the NetNES 1.1 server. (C) Pax7 has NLS residues from 134 to 220 that are a potential site for importin to bind to, indicating that Pax7 is a nuclear protein that is translocated into the nucleus. (D) The NLS sequence highlighting the predicted residues for phosphorylation as analysed using the NetPhos 2.0 server.

\section{A}

Sp|Q02650|PAX5_MOUSE Paired box protein Pax-5 OS=Mus musculus $G N=P$ ax5 $P E=1$ SV=1 Sequence ID: ICl|61301 Length: 391 Number of Matches: 2

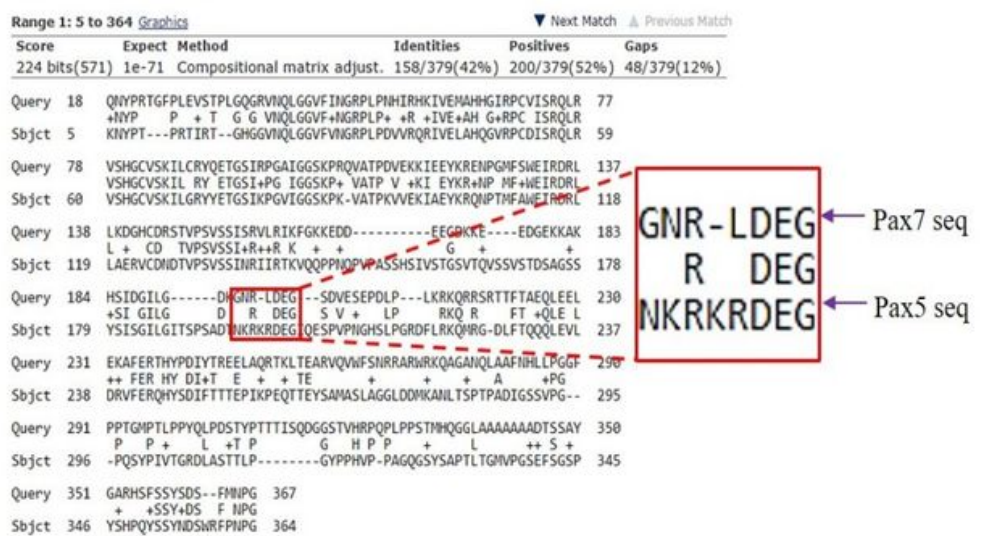

B

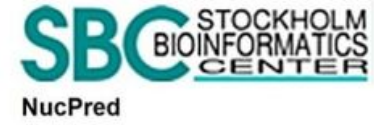

The NucPred score for your sequence is 0.80 (see score help below
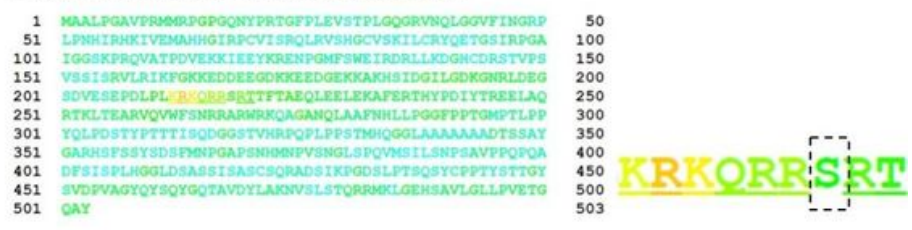

NucPred
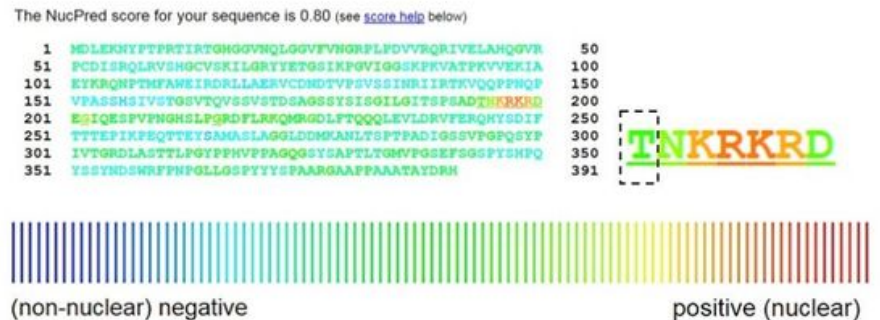

\section{Figure 5}

Prediction of Importin-a1 and Importin13 as Pax7 Translocator in Myoblasts (A) Alignment of Pax5 and Pax7 sequences. The alignment shows that only 4 out of 8 Pax7 residues are similar to the Pax 5 residue binding site. Alignment of the two sequences was performed using blastp algorithm from the BLAST website. (B) NLS analysis of the Pax7 sequence compared to the Pax5 sequence. Analysis of the NLS using NucPred by Brameier et al., 2007. The 'S' (serine) 
and ' $T$ ' (Threonine) are residue which are expected to be phosphorylated based on the prediction phosphorylation analysis using NetPhos2.0. (https://www.sbc.su.se/ maccallr/nucpred/cgi-bin/single.cgi)
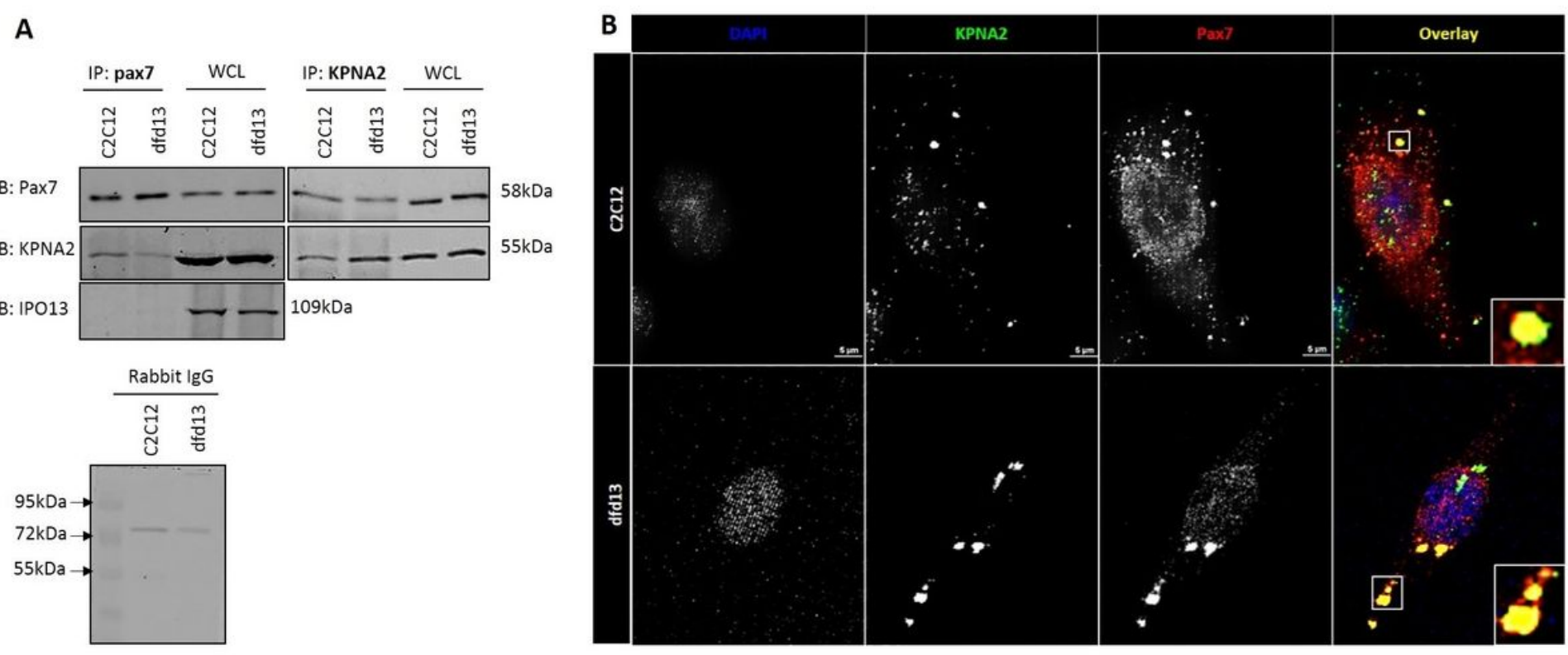

\section{Figure 6}

Endogenous Pax7 associates and co-localised with KPNA2 (A) Approximately $500 \mu \mathrm{g}$ of whole cell lysates of C2C12 and dfd13 myoblasts were used for immunoprecipitation analysis. Endogenous Pax7 was pulled down using a protein A sepharose CL-4B bead slurry, and KPNA2 was pulled down using Dynabeads Protein $\mathrm{G}$ magnetic beads. Both purified proteins were blotted with anti-Pax7, KPNA2 and IP013 antibodies. Whole cell lysate was used as a positive control and rabbit IgG (with sample buffer) as the negative control. (B) Approximately $1.5 \times 104$ C2C12 and dfd13 myoblasts were cultured in GM in a 48-well plate overnight. The myoblasts were fixed in 4\% paraformaldehyde prior to immunolabelling with for Pax7 and KPNA2. DAPI was used as a nuclear counterstain. The images were captured using a confocal microscope Nikon 1AR. Co-localisation analysis has performed using NIS element software provided by Nikon. GM: growth medium (DMEM + 10\% FCS); $n=3$. 